\title{
A novel animal model for bone metastasis in human lung cancer
}

\author{
MI LI ${ }^{1}$, MINGLIANG ZHOU ${ }^{1}$, MENG GONG ${ }^{1,2}$, JUNRONG MA ${ }^{1,2}$, FUXING PEI $^{4}$, WESLEY G. BEAMER ${ }^{3}$, \\ LEONARD D. SHULTZ ${ }^{3}$, JANET M. HOCK ${ }^{2}$ and XIJIE YU ${ }^{1,2}$
}

\author{
${ }^{1}$ Laboratory of Endocrinology and Metabolism, West China Hospital, Sichuan University, Chengdu, Sichuan 610041, \\ P.R. China; ${ }^{2}$ Maine Institute for Human Genetics and Health, Bangor, ME; ${ }^{3}$ The Jackson Laboratory, Bar Harbor, ME, USA; \\ ${ }^{4}$ Department of Orthopaedic Surgery, West China Hospital, Sichuan University, Chengdu, Sichuan 610041, P.R. China
}

Received November 23, 2011; Accepted January 20, 2012

DOI: $10.3892 / \mathrm{ol} .2012 .586$

\begin{abstract}
Metastases account for $90 \%$ of lung cancer mortalities, frequently target the skeleton and lead to rapid deterioration in quality of life. The molecular mechanism underlying bone metastases is largely unknown. Development of xenograft mouse models, such as the severe combined immunodeficient (SCID) CB-17 mouse and the non-obese diabetic (NOD)/SCID mouse, both of which lack functional Band T-cells and are able to host allogeneic or xenogeneic tumor cells, has made great contributions in this area. However, residual natural killer (NK) cells in these models are able to significantly modify local tumor growth and metastasis. Treatment with anti-murine IL-2 receptor $\beta$ chain $\mathrm{Ab}$ (TM- $\beta 1$ ) antibody can abrogate NK cell activity in vivo; however, the antibody treatment may result in unexpected effects and the stability is hard to control. To overcome these shortcomings, we evaluated xenografts in NOD-scid IL2R $\gamma^{\text {null }}$ immunodeficient mice that lacked mature $\mathrm{T}$ cells, $\mathrm{B}$ cells and functional NK cells. We compared the target tissue distribution of the human small cell lung cancer cell lines SBC-5 and SBC-3. Gross necropsy and whole skeletal X-ray film examination of the host mice were conducted 30 days post-tail vein injection. The SBC-5 cells colonized bone and formed lytic lesions. The cells also colonized liver, spleen and, less frequently, the pancreas, ovary and kidney. The SBC-3 cell xenografts formed easily visible tumor foci in the liver, pancreas, ovary/uterus and kidney, but not bone metastases. Our results showed that SBC-5 cells in NOD-scid IL2R $\gamma^{\text {null }}$ immunodeficient mice provide a suitable xenograft model system for bone metastasis of human lung cancer. This novel animal model may therefore be used to study the molecular pathway of bone metastases and to evaluate targets for effective therapies.
\end{abstract}

Correspondence to: Dr Xijie Yu, Laboratory of Endocrinology and Metabolism, West China Hospital, Sichuan University, No. 37 Guoxue Xiang, Chengdu, Sichuan 610041, P.R. China

E-mail: xijieyu@hotmail.com

Key words: lung cancer, bone metastasis, animal model

\section{Introduction}

Lung cancer is the major cause of malignancy-related mortality worldwide, and its incidence is on the rise in many countries (1). Despite successful treatment of the primary malignancy, relapse and subsequent metastatic spread can still occur at distant sites, including bone, liver and brain, accounting for $90 \%$ of lung cancer mortalities (1). Over one third of patients with advanced lung cancer are reported to develop osteolytic bone metastases, which cause pathological fractures, disability, pain, nerve compression (including spinal cord compression), anemia and hypercalcemia, leading to rapid deterioration in quality of life $(2,3)$. The median survival time (MST) of patients with such metastases is 6-7 months (3). Although important advances have been made in understanding the pathogenesis and treatment of the primary malignancy, little progress has been made in treating skeletal metastasis. Bone complications often require surgery to correct fractures or spinal deformities, radiation therapy to control the severe pain and/or the use of bisphosphonates. Unfortunately, there has not been significant long-term success for patients who are treated with bisphosphonates following clinical diagnosis of lung cancer $(4,5)$.

Bone metastases occur as a sequence of multi-step events regulated by cancer cells and host microenvironments. The majority of bone lesions associated with lung cancer metastases are osteolytic, producing or inducing agents and factors that act in a paracrine manner to activate osteoclasts, leading to bone resorption and destruction $(6,7)$. Bone resorption is accompanied by the release of growth factors and cytokines previously deposited within the matrix during bone formation. These growth factors act, in turn, back on the lung cancer cells to promote their further proliferation, establishing a 'vicious cycle' of lung cancer skeletal metastasis $(6,8)$.

The study of cancer metastasis in humans, particularly bone metastasis, is challenging due to the limited access to the sites of tumor growth, ethical concerns and the confounding effects of therapy on tumor growth and biology (9). To overcome these restrictions, human tumor growth and metastasis have been evaluated in a variety of SCID mouse models. Challenges to human-cell engraftment in CB17-SCID mice include the spontaneous generation of mouse $\mathrm{T}$ and $\mathrm{B}$ cells during aging (known as leakiness) and high levels of host natural killer 
(NK)-cell and other innate immune activity (9). NK cells are crucial in the rejection of implanted tissues or cells in SCID mice $(10,11)$. Thus repeated transplantation, long periods of time, total body irradiation of mice, hormone supplements, etoposide pretreatment or NK monoclonal antibodies (mAbs) are required for consistent and reproducible tumor formation. Depending on the type of treatment, immunosuppressive conditioning releases a cascade of pro-inflammatory cytokines that modify the activity of tumor stroma, such as fibroblasts, myoepithelial cells and macrophages, which interact functionally with adjacent tumor cells $(11,12)$. The CB17-SCID mutation also results in defective DNA repair and, consequently, an increase in radiosensitivity (13), which may affect the success of xenografts. In comparison with conventional SCID, the NOD-SCID strain appears to be more promising as a tool for xenotransplantion of human tumors due to the lower NK-cell activity. However, the use of NOD-SCID mice as a model for human tumor metastasis remains limited by their relatively short life span, the residual activity of NK cells, macrophage function, complement activity and the function of dendritic cells (11). In addition, residual dendritic cells are important in transplant rejection (14). The aim of the present study was to establish a novel animal model for bone metastasis of human lung cancer with NOD scid/scid IL-2ry -/- immunodeficient mice that lack mature T cells, B cells and functional NK cells.

\section{Materials and methods}

Human SCLC cell lines. Human SBC-5 and SBC-3 small cell lung cancer (SCLC) cell lines were obtained from the Japan Health Sciences Foundation, Health Science Resources Bank (HSRB; JCRB0819 and JCRB0818). The two cell lines were cultured in $25 \mathrm{~cm}^{2}$ Falcon culture flasks in $\alpha$-minimal essential medium ( $\alpha$-MEM; Invitrogen, Grand Island, NY, USA) with $10 \%$ fetal calf serum (Invitrogen) and $1 \%$ penicillin-streptomycin solution (Invitrogen) at $37^{\circ} \mathrm{C}$ with $5 \% \mathrm{CO}_{2}$. Medium was changed every 2 days. To obtain sufficient numbers of cells for in vivo studies, cells were briefly trypsinized, centrifuged and washed 2 times in serum-free medium, counted and then resuspended in PBS for tail vein injection.

Mice. NOD-scid IL $2 R \gamma^{\text {null }}$ mice were raised in our research colonies (LDS) at The Jackson Laboratory, Bar Harbor, ME, USA. Mice were housed under barrier conditions in groups of 2-4 same sex mice within polycarbonate cages $\left(324 \mathrm{~cm}^{2}\right)$. Colony environmental conditions included 14:10-h light:dark cycles, sterilized white pine shavings for bedding, NIH 31 irradiated diet (6\% fat, $19 \%$ protein, $\mathrm{Ca}: \mathrm{P}$ of $1.15: 0.85)$ and vitamin and mineral fortification (Purina Mills International, Brentwood, MO, USA), with sterilized water ad libitum. All procedures involving mice were reviewed and approved by the Institutional Animal Care and Use Committee.

Xenograft of human SCLC cell lines. SBC-5 was selected for the present study as it was the only small-cell carcinoma cell line of several lines tested (SBC-5, SBC-3, SBC-3/ADM, H69, H69/ VP) that formed bone metastases in NK-cell-depleted SCID mice (15). The SBC-5 and SBC-3 cells colonize abdominal organs and lungs of various mouse genetic background hosts homozygous for severe combined immunodeficiency (scid/scid) that have been treated with anti-IL-2r $\gamma$ sera (16). SBC-5 cells are unique in that they also colonize skeletal tissue sites (17).

Our in vivo studies were conducted with NOD-scid $I L 2 R \gamma^{\text {null }}$ host mice injected via the tail vein with $1.0 \times 10^{6}$ cells in PBS. The age of mice ranged from 8 to 14 weeks, with age being systematically balanced among the groups. Daily observation of host mice revealed clear signs of physiological distress 28-36 days following treatment. Accordingly, mice were sacrificed on day 30. Representative abdominal organs showing metastatic colonies and all lungs were fixed in $10 \%$ neutral-buffered formalin for histological analyses. Whole body skeletons were X-rayed to detect the presence of bone lytic lesions using a Faxitron MX20 cabinet instrument, and high resolution images were captured on Kodak MIN-R 2000 mammography film. Representative bones with identified bone lytic lesions were retained for histological study.

Histology. Liver and bone with metastatic colonies from NOD-scid IL $2 R \gamma^{\text {null }}$ recipients were selected for routine histological examination. Liver and bone were fixed in $10 \%$ neutral-buffered formalin for $48 \mathrm{~h}$; bone was further decalcified with $10 \%$ EDTA for $12 \mathrm{~h}$. Tissues were processed for embedding in paraffin wax and sectioning. Paraffin-embedded sections were stained with hematoxylin and eosin (H\&E) and visualized under a Zeiss Axiovert 200M microscope.

\section{Results}

Distribution of bone lytic lesions only in SBC-5 hosts. NOD-scid $I L 2 R \gamma^{\text {null }}$ mice were injected by the tail vein with either SBC-5 or SBC-3 cells at a concentration of $1 \times 10^{6}$ per mouse. The mice were sacrificed on day 30 following injection. Mice (20/20) receiving SBC-5 cells had bone metastases as shown by X-ray analysis (Fig. 1; Table I). No bone metastases were found in mice receiving SBC-3 cells (0/9, data not shown). As with osteolytic bone metastases in human lung cancer, the bone metastases in SBC-5 host mice were bone lytic lesions (Fig. 1). Multiple bone lytic lesions were detected in all mice with SBC5 xenografts (Fig. 1). Bone lytic lesions were mostly located in the femur (18/20) and vertebrae (20/20). The high frequency of bone lytic lesions in tails $(14 / 20)$ may be due to cell leakage as the injection needle is withdrawn. Histological analysis showed that metastatic colonies were located within cancellous bone (Fig. 2). Metastatic tumor xenografts were surrounded by bone marrow cells (Fig. 2). The results from $\mathrm{X}$-ray and histological analyses indicate that the bone lytic lesions are similar to the osteolytic bone metastases found in human lung cancer.

Similar metastases in other organs between SBC-5 and $S B C-3$ hosts. Besides the bone lytic lesions, SBC-5 xenografts in NOD-scid IL $2 R \gamma^{\text {null }}$ host mice developed metastases in the liver, spleen and, less frequently, the pancreas, ovary and kidney. The SBC-3 cell xenografts formed easily visible tumor foci in the liver, pancreas, ovary/uterus and kidney. There was no marked difference in soft tissue metastatic locations and gross shape in mice with SBC-5 and SBC-3 xenografts (data not shown). Neither SBC-5 nor the SBC-3 SCLC cells colonized mouse lung tissue. All lungs from mice with either SBC-5 or SBC-3 xenografts were free of histologically identi- 
Table I. Summary of bone sites showing lytic lesions detected by whole body X-ray analyses.

\begin{tabular}{lcccccccc}
\hline & & & \multicolumn{3}{c}{ Vertebrae } & & & \\
Bone & Femur & Tibia & Spine & Sacrum & Tail & Forelimb & Ileum & Other sites \\
\hline Female & $7 / 7$ & $5 / 7$ & $6 / 7$ & $4 / 7$ & $5 / 7$ & $1 / 7$ & $3 / 7$ & $2 / 7$ \\
Male & $11 / 13$ & $5 / 13$ & $2 / 13$ & $8 / 13$ & $9 / 13$ & $0 / 13$ & $2 / 13$ & $1 / 13$ \\
Female + male & $18 / 20$ & $10 / 20$ & $8 / 20$ & $12 / 20$ & $14 / 20$ & $1 / 20$ & $5 / 20$ & $3 / 20$ \\
\hline
\end{tabular}

Whole body skeletons of the SBC-5 hosts were X-rayed to dectect the presence of bone lytic lesions using a Faxitron MX20 cabinet instrument and high resolution images were captured on Kodak MIN-R 2000 mammography film. The bone lytic lesions in each mouse were counted and summarized.
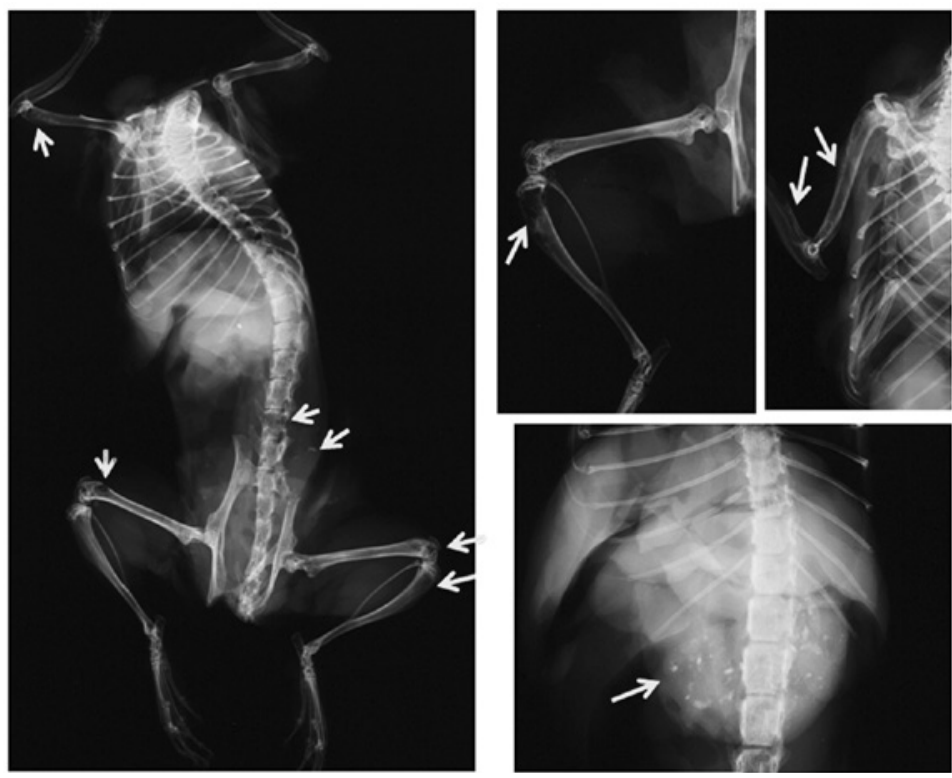

Figure 1. X-ray analysis of bone metastases. NOD-scid IL2R $\gamma^{\text {null }}$ host mice were injected with $1.0 \times 10^{6} \mathrm{SBC}-5$ cells via the tail vein. The mice were sacrificed 30 days following injection. Whole body skeletons were X-rayed to detect the presence of bone lytic lesions using a Faxitron MX20 cabinet instrument, and high resolution images were captured on Kodak MIN-R 2000 mammography film. Bone lytic lesions were detected in each mouse. The major lesions were located in the femur, tibia and vertebrae (arrow). Some high density dots were found in the liver (arrow).

fied tumor foci (data not shown). Livers from mice with SBC-5 xenografts contained large numbers of tumor cell nodules ranging from 2 to $4 \mathrm{~mm}$ in diameter (Fig. 3B). Multiple metastatic colonies were detected in the liver of mice with SBC-5 xenografts, (Fig. 3C and D). Although microcalcifications were observed in the livers of some mice with SBC-5 xenografts (Fig. 1), calcification was not detected by histology due to decalcification processing (Fig. 3C and D).

\section{Discussion}

An obstacle to better understanding the mechanisms of lung cancer metastasis is the lack of reproducible animal models of the biology of lung cancer metastasis to bone. An early animal model system utilized to investigate engraftment and proliferation of normal and neoplastic human cells was the severe combined immunodeficiency (SCID) CB17 mouse. This mouse lacks functional $\mathrm{B}$ and $\mathrm{T}$ cells and can accept allogeneic or xenogeneic tumor cell grafts (18), but these xenografts may not reproduce the required metastatic lung cancer phenotype, and consistency in outcomes may be difficult to achieve. Although lung cancer cell line xenografts may survive in SCID mice, pretreatment of mice with anti-GM1 serum, and depletion of macrophages and NK cells, is necessary for complete immunosuppression (19), and only tumors that have already metastasized in the human patient are likely to grow in the SCID mouse model (20). Tumor xenografts typically lack the growth characteristics and metastatic properties of human lung cancer cell xenografts in SCID mice. The nonobese diabetic (NOD)/LtSz scid/scid (NOD/SCID) mouse is the most immunodeficient of SCID variants, as it lacks functional B and T cells and circulating complement $(9,21)$. Human fetal bone and, recently, adult human rib have been implanted into NOD/SCID mice, a model termed NOD/SCID-hu, and utilized to study prostate, breast and lung cancer metastatic effects on bone (22-25). However, residual NK cells in these models may alter tumor growth in bone, and metastasis due to direct cell cytotoxicity without prior sensitization and secretion of immunostimulatory cytokines, such as, IFN- $\gamma$ is not suppressed $(10,26)$. Treatment with anti-murine IL-2 receptor 

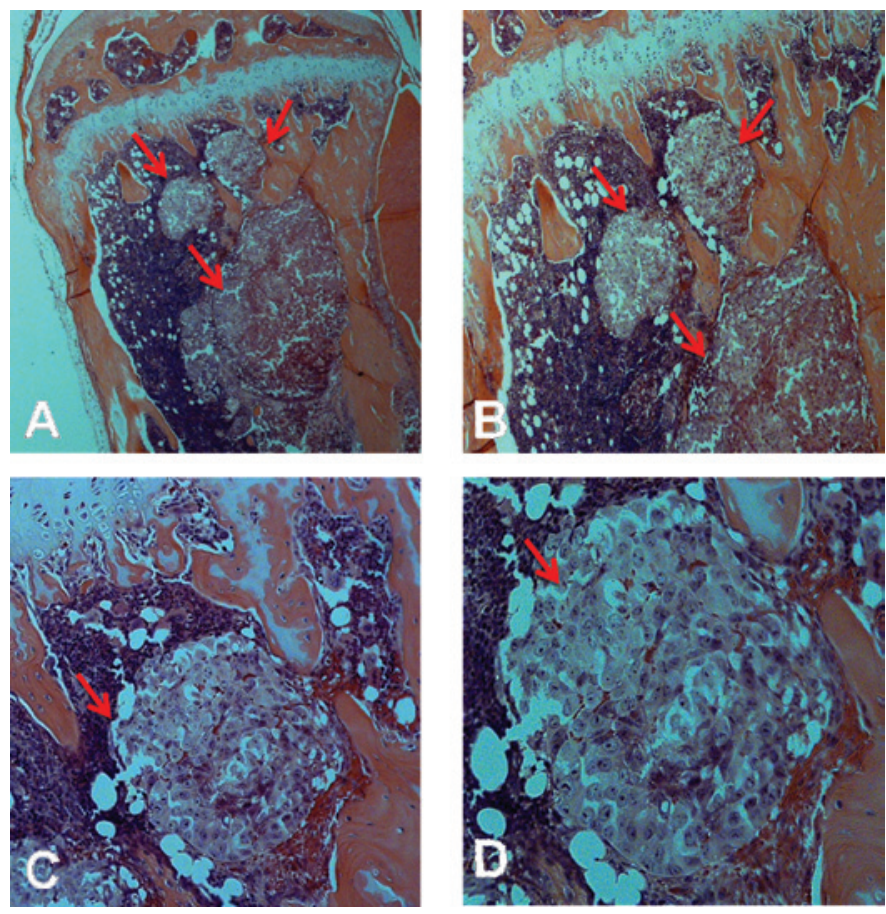

Figure 2. Histological analysis of bone metastases. NOD-scid IL2R $\gamma^{\text {null }}$ host mice were injected with $1.0 \times 10^{6}$ SBC-5 cells via the tail vein. The mice were sacrificed 30 days following injection. A tibia with metastatic tumors was fixed in $10 \%$ neutral-buffered formalin for $48 \mathrm{~h}$, and further decalcified with $10 \%$ EDTA for $12 \mathrm{~h}$. Paraffin-embedded sections were stained with H\&E staining and visualized under the microscope. Three metastatic colonies were detected in the bone marrow cavity (arrows in A and B). (A-C) The metastatic colonies were surrounded by bone marrow cells. (C) One colony was located close to the growth plate. (D) The metastatic colony was enriched with tumor cells (magnification: A, x50; B, x100; C, x200; D, x400). H\&E, hematoxylin and eosin.
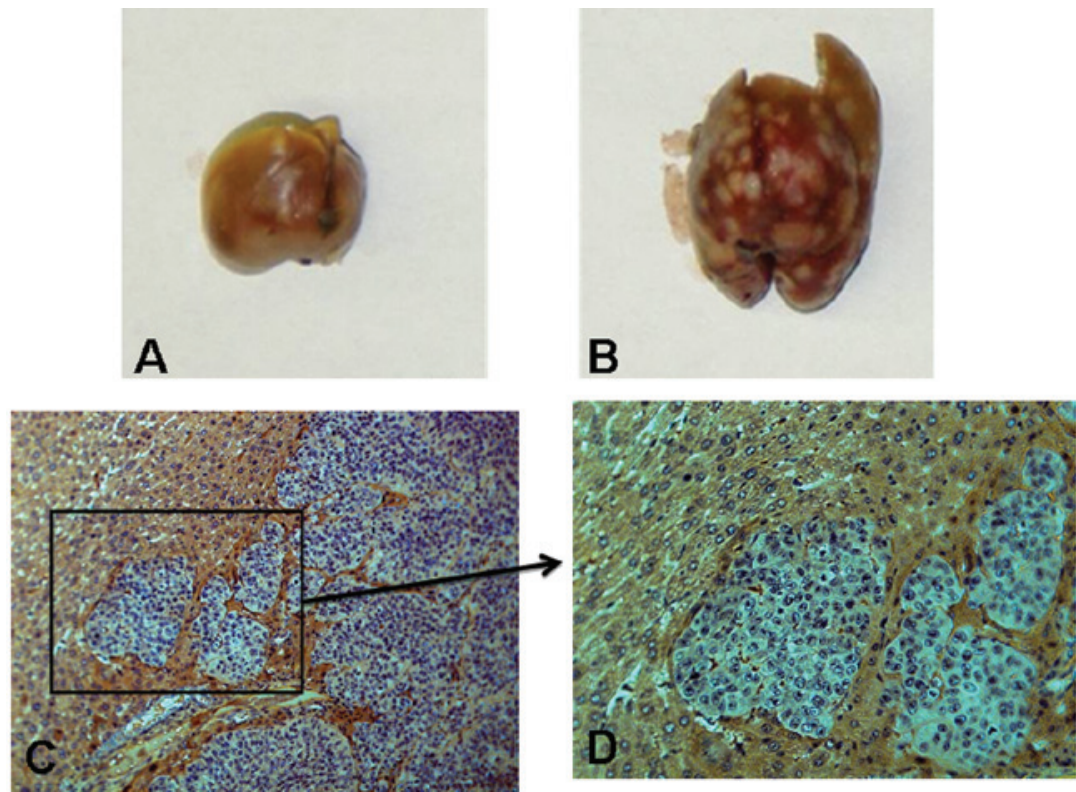

Figure 3. Histological analysis of liver metastases. NOD-scid IL2R $\gamma^{\text {null }}$ host mice were injected with $1.0 \times 10^{6}$ SBC-5 cells via the tail vein. The mice were sacrificed 30 days following injection. A liver with metastatic tumors was fixed in 10\% neutral-buffered formalin for $48 \mathrm{~h}$. Tissues were processed for embedding in paraffin wax, and were sectioned and stained with H\&E. (A) The liver from the control mouse. (B) The liver from the SBC-5 host showed large numbers of nodules ranging from 2 to $4 \mathrm{~mm}$ in diameter. (C and D) Multiple metastatic colonies were detected in the liver from the SBC-5 host, the metastatic colony was enriched with tumor cells (magnification: C, x50; D, x200). H\&E, hematoxylin and eosin.

$\beta$ chain Ab (TM- $\beta 1)$ antibody abrogates NK-cell activity in vivo (15); however, this antibody treatment may induce unexpected effects and the phenotype induced is inconsistent.

A novel immunodeficiency mouse strain was recently developed by Dr L. Shultz at the Jackson Laboratory (JAX).
This mutant mouse combines the features of the NOD/ShiLtJ background, the SCID and IL2 receptor $\gamma$ chain deficiency (9). As a result, the NOD.Cg-Prkdcscid Il2rgtm1Wjl/SzJ mice do not express the Prkdc gene or the X-linked IL2 receptor $\gamma$ chain gene. Histological examination of lymphoid tissues 
reveals the absence of lymphoid cells and certain cystic structures in the thymus, an absence of follicles in the spleen and markedly diminished cellularity of lymph nodes (9). NOD-scid IL2R $\gamma^{\text {null }}$ mice are deficient in mature lymphocytes, serum Ig is not detectable and NK-cell cytotoxic activity is markedly low. The lack of mature T cells, B cells or functional NK cells, and deficiency in cytokine signaling lead to better engraftment of human tumor cell lines and human primary tumors (9). NOD-scid IL2R $\gamma^{\text {null }}$ mice have been widely used in islet transplantation and studies of hematopoietic stem cells and cancer stem cells.

We demonstrated that NOD-scid IL2R $\gamma^{\text {null }}$ immunodeficient mice provide a suitable model system for studies of metastatic human small cell lung cancer. This immunodeficient mouse strain is homozygous for targeted mutations at the IL-2R $\gamma$-chain locus $(9,27)$, which is a crucial component of the high-affinity receptors for IL-2, IL-4, IL-7, IL-9, IL-15 and IL-21, and required for signaling through these receptors (28). Absence of the IL-2R $\gamma$-chain leads to severe impairment of Tand B-cell development and function, and completely prevents NK-cell development (29-31). Our observations show that the two human small cell lung cancer cell lines, SBC-3 and SBC-5, inoculated in NOD scid/scid Il-2ry -/- immunodeficient mice form tumor foci in multiple organs, while maintaining their common histomorphology. Only SBC-5 cells colonized bone with lytic lesions. Based on these results, NOD-scid $I L 2 R \gamma^{\text {null }}$ immunodeficient mice with SBC-5 and/or SBC-3 xenografts can be applied to studies of metastasis, particularly bone metastasis, of human small cell lung cancer. This novel animal model using SBC-5 or SBC-3 xenografts in NOD-scid $I L 2 R \gamma^{\text {null }}$ immunodeficient mice may be used in studies of the molecular pathways of human small cell lung cancer skeletal metastases and to evaluate targets for effective therapies.

\section{Acknowledgements}

This study was supported by grants to Dr Xijie Yu from National Natural Science Foundation of China (No. 81072190), and US Dept Defense Telemedicine and Technology Research Command Award W81XWH-07-2-0116 (PI JM Hock). Some experiments were performed by Drs X. Yu, M. Gong and Ms J. Ma while employed at the Maine Institute for Human Genetics and Health.

\section{References}

1. Quint LE, Francis IR, Wahl RL and BH G: Imaging of lung cancer In: Lung Cancer, Principles and Practice. Lippincott-Raven, Philadelphia, pp775-789, 1996.

2. Mundy GR: Metastasis to bone: causes, consequences and therapeutic opportunities. Nat Rev Cancer 2: 584-593, 2002.

3. Mantyh PW: Cancer pain and its impact on diagnosis, survival and quality of life. Nat Rev Neurosci 7: 797-809, 2006.

4. Rubens RD: The nature of metastatic bone disease. In: Bone Metastases: Diagnosis and Treatment. Rubens RD, Fogelman I (eds.). Springer-Verlag, New York, pp1-9, 1991.

5. Diel IJ, Solomayer EF and Bastert G: Treatment of metastatic bone disease in breast cancer: bisphosphonates. Clin Breast Cancer 1: 43-51, 2000.

6. Casimiro S, Guise TA and Chirgwin J: The critical role of the bone microenvironment in cancer metastases. Mol Cell Endocrinol 310: 71-81, 2009.

7. Roodman GD: Biology of osteoclast activation in cancer. J Clin Oncol 19: 3562-3571,2001.
8. Reddi AH, Roodman D, Freeman C and Mohla S: Mechanisms of tumor metastasis to the bone: challenges and opportunities. J Bone Miner Res 18: 190-194, 2003.

9. Shultz LD, Ishikawa F and Greiner DL: Humanized mice in translational biomedical research. Nat Rev Immunol 7: 118-130, 2007.

10. Wu J and Lanier LL: Natural killer cells and cancer. Adv Cancer Res 90: 127-156, 2003.

11. Dewan MZ, Terunuma H, Ahmed S, et al: Natural killer cells in breast cancer cell growth and metastasis in SCID mice. Biomed Pharmacother 59 (Suppl 2): 375-379, 2005.

12. Quarmby S, Kumar P and Kumar S: Radiation-induced normal tissue injury: role of adhesion molecules in leukocyte-endothelial cell interactions. Int J Cancer 82: 385-395, 1999.

13. Fulop GM and Phillips RA: The scid mutation in mice causes a general defect in DNA repair. Nature 347: 479-482, 1990.

14. Lechler R, Ng WF and Steinman RM: Dendritic cells in transplantation--friend or foe? Immunity 14: 357-368, 2001

15. Yano S, Nishioka Y, Izumi K, et al: Novel metastasis model of human lung cancer in SCID mice depleted of NK cells. Int J Cancer 67: 211-217, 1996.

16. Yano $\mathrm{S}$ and Sone S: Novel metastasis model of human lung cancer cells representing different histological types in SCID mice depleted of NK cells. Gan To Kagaku Ryoho 24: 489-494, 1997.

17. Miki T, Yano S, Hanibuchi M and Sone S: Bone metastasis model with multiorgan dissemination of human small-cell lung cancer (SBC-5) cells in natural killer cell-depleted SCID mice. Oncol Res 12: 209-217, 2000.

18. Bosma GC, Custer RP and Bosma MJ: A severe combined immunodeficiency mutation in the mouse. Nature 301: 527-530, 1983.

19. Yang W, Lam P, Kitching R, et al: Breast cancer metastasis in a human bone NOD/SCID mouse model. Cancer Biol Ther 6: 1289-1294, 2007.

20. Murthy MS, Scanlon EF, Jelachich ML, Klipstein S and Goldschmidt RA: Growth and metastasis of human breast cancers in athymic nude mice. Clin Exp Metastasis 13: 3-15, 1995.

21. Shultz LD, Schweitzer PA, Christianson SW, et al: Multiple defects in innate and adaptive immunologic function in NOD/ LtSz-scid mice. J Immunol 154: 180-191, 1995.

22. Shtivelman E and Namikawa R: Species-specific metastasis of human tumor cells in the severe combined immunodeficiency mouse engrafted with human tissue. Proc Natl Acad Sci USA 92: 4661-4665, 1995

23. Nemeth JA, Harb JF, Barroso U Jr, He Z, Grignon DJ and Cher ML: Severe combined immunodeficient-hu model of human prostate cancer metastasis to human bone. Cancer Res 59: 1987-1993, 1999.

24. Yonou H, Yokose T, Kamijo T, et al: Establishment of a novel species- and tissue-specific metastasis model of human prostate cancer in humanized non-obese diabetic/severe combined immunodeficient mice engrafted with human adult lung and bone. Cancer Res 61: 2177-2182, 2001.

25. Kuperwasser C, Dessain S, Bierbaum BE, et al: A mouse model of human breast cancer metastasis to human bone. Cancer Res 65: 6130-6138, 2005.

26. Shankaran V, Ikeda H, Bruce AT, et al: IFNgamma and lymphocytes prevent primary tumour development and shape tumour immunogenicity. Nature 410: 1107-1111, 2001

27. Ito M, Hiramatsu H, Kobayashi K, et al: NOD/SCID/gamma(c) (null) mouse: an excellent recipient mouse model for engraftment of human cells. Blood 100: 3175-3182, 2002.

28. Sugamura K, Asao H, Kondo M, et al: The interleukin-2 receptor gamma chain: its role in the multiple cytokine receptor complexes and $\mathrm{T}$ cell development in XSCID. Annu Rev Immunol 14: 179-205, 1996

29. Cao X, Shores EW, Hu-Li J, et al: Defective lymphoid development in mice lacking expression of the common cytokine receptor gamma chain. Immunity 2: 223-238, 1995.

30. DiSanto JP, Muller W, Guy-Grand D, Fischer A and Rajewsky K: Lymphoid development in mice with a targeted deletion of the interleukin 2 receptor gamma chain. Proc Natl Acad Sci USA 92: 377-381, 1995.

31. Ohbo K, Suda T, Hashiyama M, et al: Modulation of hematopoiesis in mice with a truncated mutant of the interleukin-2 receptor gamma chain. Blood 87: 956-967, 1996. 